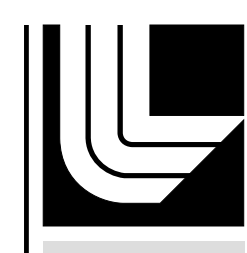

LA W RENCE LIVERM ORE NATIONAL LABORATORY

\title{
Ultrafast observation of shocked states in a precompressed material
}

M. R. Armstrong, J. C. Crowhurst, S. Bastea, J. M. Zaug

July 15,2010

Journal of Applied Physics 
This document was prepared as an account of work sponsored by an agency of the United States government. Neither the United States government nor Lawrence Livermore National Security, LLC, nor any of their employees makes any warranty, expressed or implied, or assumes any legal liability or responsibility for the accuracy, completeness, or usefulness of any information, apparatus, product, or process disclosed, or represents that its use would not infringe privately owned rights. Reference herein to any specific commercial product, process, or service by trade name, trademark, manufacturer, or otherwise does not necessarily constitute or imply its endorsement, recommendation, or favoring by the United States government or Lawrence Livermore National Security, LLC. The views and opinions of authors expressed herein do not necessarily state or reflect those of the United States government or Lawrence Livermore National Security, LLC, and shall not be used for advertising or product endorsement purposes. 


\title{
Ultrafast observation of shocked states in a precompressed material
}

Michael R. Armstrong*, Jonathan C. Crowhurst, Sorin Bastea, and Joseph M. Zaug

Physical and Life Sciences Directorate, Lawrence Livermore National Laboratory, Livermore, California 94550, USA

*email: armstrong30@llnl.gov

\begin{abstract}
We apply ultrafast single shot interferometry to determine the pressure and density of argon shocked from up to $7.8 \mathrm{GPa}$ static initial pressure in a diamond anvil cell. This method enables the observation of thermodynamic states distinct from those observed in either single shock or isothermal compression experiments. In particular, this method enables access to high density, relatively low temperature states of light materials, such as isentropically compressed states of giant planets. Further, since excitation by a shock wave is intrinsically ultrafast and this method has picosecond time resolution, it has the potential to observe the collective dynamics of materials undergoing shock induced phase transitions and chemistry on ultrafast time scales. We also present a straightforward method for interpreting ultrafast shock wave data which determines the index of refraction at the shock front, and the particle and shock velocities for shock waves in transparent materials. Based on these methods, we observe shocked thermodynamic states between the room temperature isotherm of argon and the shock adiabat of cryogenic argon at final shock pressures up to $28 \mathrm{GPa}$.
\end{abstract}




\section{Introduction}

For decades, compression experiments have been used to determine thermodynamic states of materials at high pressure and temperature. Such data are necessary to correctly interpret seismic data ${ }^{1,2}$, understand planetary composition ${ }^{3}$, the evolution of the early solar system ${ }^{4}$, shock wave induced chemistry ${ }^{5}$, and fundamental issues in condensed matter physics ${ }^{6-8}$. Most compression experiments have applied either static compression in a diamond anvil cell (DAC) ${ }^{9-13}$, or dynamic compression using shock waves ${ }^{6}$. Broadly, these methods obtain material properties along two onedimensional trajectories in thermodynamic phase space - the isotherm (via static compression) and the shock adiabat, or Hugoniot. Although highly dependent on the sample and the specific method, generally DAC methods obtain relatively low temperature, isothermal compression at less than $~ 400$ GPa pressure. In contrast, single shock methods have obtained much higher pressure (well into the TPa range), typically at much higher temperature. Although such experiments provide a wide range of material data, special techniques are required to obtain information about intermediate pressures and temperatures ${ }^{6,9,14,15}$. In particular, for single shock compression of highly compressible materials from ambient pressure, the density approaches a constant with increasing shock pressure ${ }^{6,15}$, limiting the application of single shock methods to low density states. Here we demonstrate the generation and characterization of shock waves on an ultrafast time scale in samples compressed in a conventional diamond anvil cell, under an initial static pressure up to $7.8 \mathrm{GPa}$. This method enables the investigation of 
compressed states off the principal Hugoniot and the room temperature isotherm, which are challenging to obtain using static or single shock wave compression.

Shock compression of a precompressed material enables two powerful experimental strategies. Single shock experiments provide equation of state information along a one-dimensional trajectory in pressure-density space: the shock adiabat, or Hugoniot. Since the Hugoniot depends on the initial thermodynamic state of the material, by varying the initial density through applied precompression it is possible to obtain different Hugoniots as a function of initial conditions. Generally, upon shock compression, a material initially at low density will heat more substantially than it would if it were initially at higher density. Thus, by isothermally precompressing the material the final temperature of the shocked state can be decreased and the final density increased, enabling the characterization of thermodynamic states off the principal Hugoniot (i.e. the Hugoniot whose initial state is the most common for the material in question $^{6}$ ). This method has previously been applied in large-scale laser shock experiments on longer time scales at precompression in the range of $\sim 1 \mathrm{GPa}^{15}$. Isothermal compression in a diamond anvil cell is a very effective way to control the initial density. For instance, solid argon changes in density by only $8 \%$ between $0 \mathrm{~K}$ and its boiling point at 1 bar. In contrast, argon under isothermal compression exhibits a $\sim 4 \mathrm{x}$ density increase from 0.1 to $33 \mathrm{GPa}^{16}$ applied pressure. 
Also, the initial state of the material can be placed, via precompression (preheating may also be used), in the proximity of a phase transition or chemical reaction boundary before shock compression, enabling the observation of phase transition or chemical dynamics as the material is shock compressed across the boundary. We note that, in cases where shock induced chemistry or phase transitions do not occur, shock compression obtains equilibrium compressed states over picosecond time scales ${ }^{6}$. When materials undergo structural changes upon compression, shock compression can be used to obtain ultrafast dynamic information from shocked metastable compressed states en route through a phase transition or chemistry. Such experiments can provide valuable information on early time states of shocked materials, such as the pre-chemistry shocked Hugoniot of reactive materials, or anomalous transient states preceding phase transitions ${ }^{17}$. Although ultrafast methods have been applied to investigate shock induced chemistry ${ }^{18,19}$ from ambient pressure, acoustics in the DAC ${ }^{20}$, and shock waves starting from ambient pressure ${ }^{21,22}$, shock waves have not been observed in precompressed materials with ultrafast time resolution.

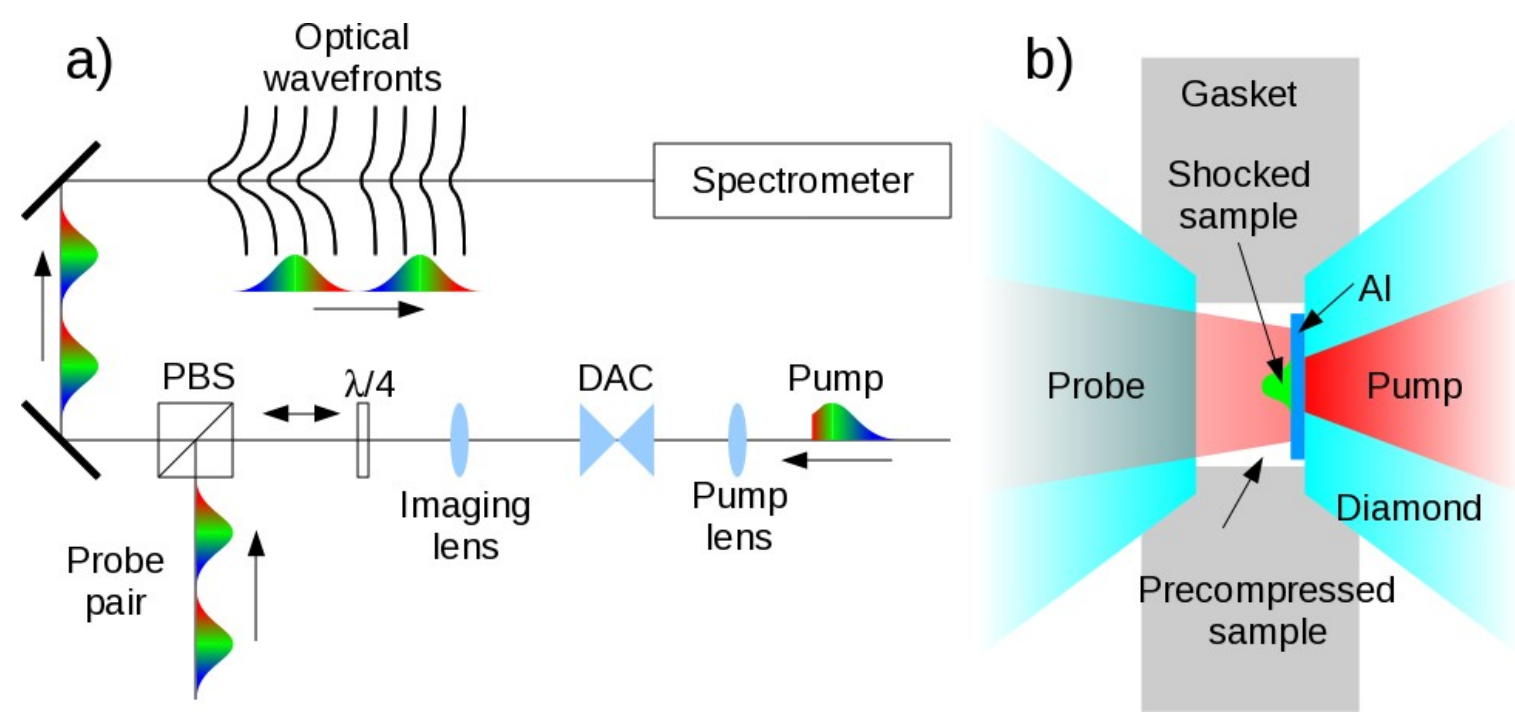

Figure 1: The experimental setup, a) shows the optical setup external to the DAC. Although the probe pulses are shown as separated for the purpose of illustration, they overlap temporally. b) shows a close up of a cross section of the DAC, where precompressed sample is shocked via ablation of a 1 micron aluminum layer on the culet of the right side diamond. PBS is a polarizing beam splitter. 
a)
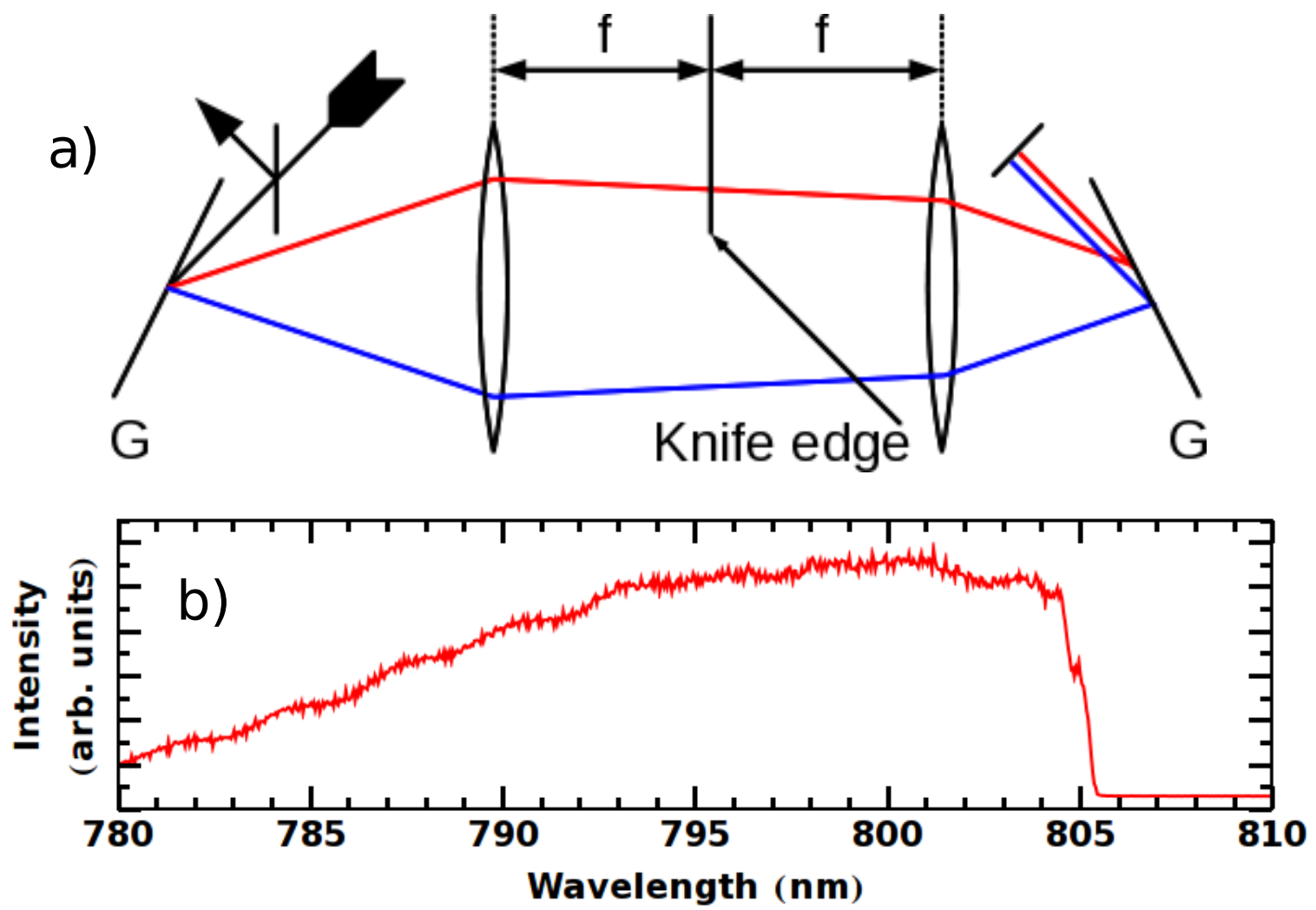

Figure 2: a) a schematic of the stretcher with a knife edge and the unblocked beam path of red and blue components of the spectrum. G labels the gratings, and $f$ is the focal length of the focusing optics. The knife edge blocks the spectrum, creating a fast rise in the intensity of the chirped pulse. b) shows a typical spectrum of the laser pulse after the amplifier.

\section{Experimental method}

An experimental schematic diagram is shown in fig. 1. In this study, the initial density of preshocked argon ranges from $1.65 \mathrm{~g} / \mathrm{cc}$ (for cryogenic liquid argon) to 2.8 g/cc (at 7.8 GPa and room temperature). To initiate shock compression of the sample, a $\sim 300$ ps, $800 \mathrm{~nm}$ center wavelength, $\sim 25 \mathrm{~nm}$ bandwidth, $\sim 100-300 \mu \mathrm{J}$ pump pulse with a less than 10 ps duration initial rise time is focused to a spot size ranging (depending on the experiment) from $20 \mu \mathrm{m}$ to $50 \mu \mathrm{m}$ FWHM intensity diameter at the diamond interface of a $\sim 1 \mu \mathrm{m}$ thick aluminum layer which partially coats one culet of one diamond in the DAC. Plasma expansion of the ablated aluminum layer drives a shock wave into the 
precompressed sample ${ }^{23}$. The laser pulse is generated by a conventional chirped pulse amplification system based on a Coherent Mira oscillator and a Legend regenerative amplifier. We use a custom built 1.5 meter stretcher with a $1800 \mathrm{~g} / \mathrm{mm}$ grating to generate 300 ps stretched pulses before amplification. The spectrum of the chirped pulse is clipped by a knife edge at the symmetry plane of the confocal imaging system in the stretcher, as shown in fig. 2a, to generate a sharp rise ( $10 \mathrm{ps})$ in the intensity profile of the chipred pulse in the time domain. Fig. 2b shows a typical spectrum after the amplifier, which is consistent in profile with previous work ${ }^{21}$.
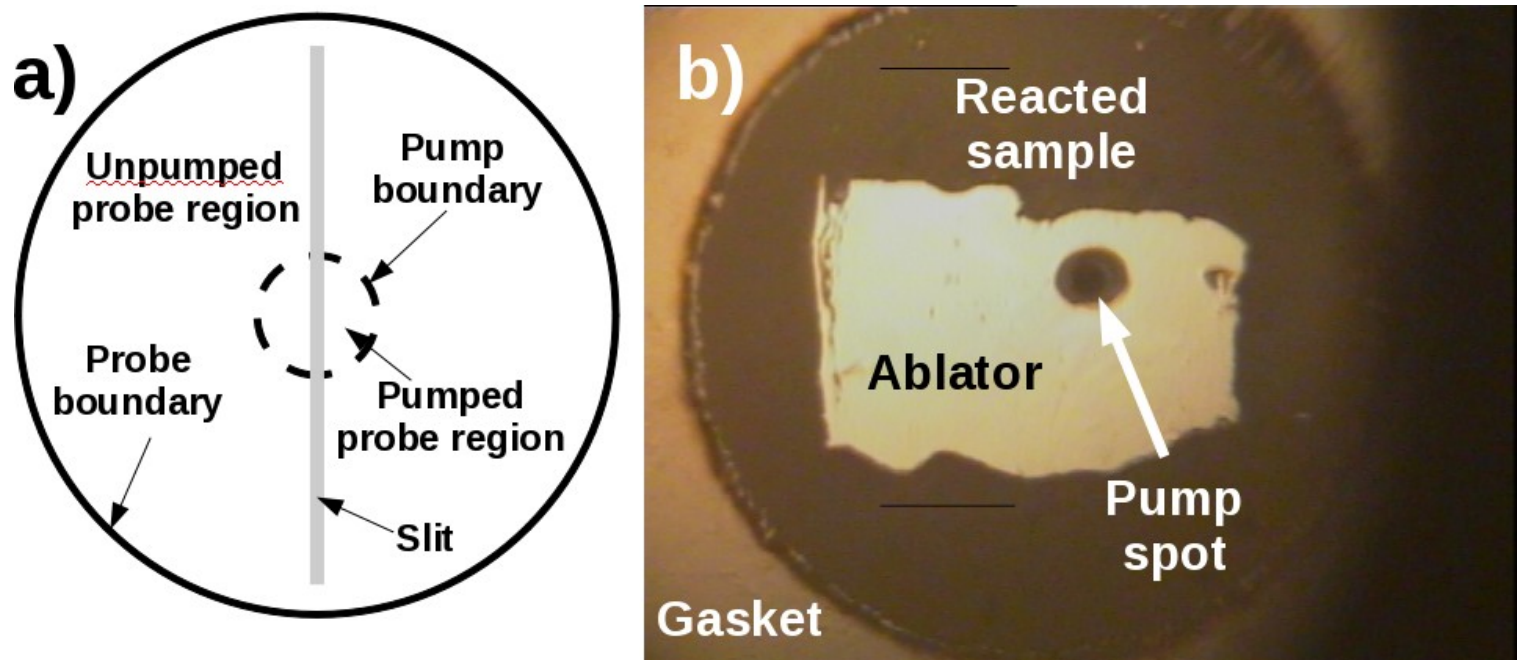

Figure 3: a) A schematic of the pump and probe imaged at the spectrometer slit and b) an image of a DAC from the pump side after an experiment. In this case, the sample was nitromethane, which reacted during the experiment and produced a black product which filled the DAC sample cavity. Although the reaction in this experiment was pump induced, it was not obviously correlated with interferometric probe data within 250 ps after the pump, and may have occurred after the probe time window. The short black horizontal line segments are camera artifacts.

The shock and particle velocities are measured via interferometry using a pair of broadband linearly chirped probe pulses (with the same spectral characteristics as the pump) separated in time by $10 \mathrm{ps}-$ a method that is the ultrafast analog to the velocity interferometry system for any reflector (VISAR) used to characterize shock waves on longer time scales ${ }^{24}$. The probe pulses are chirped to provide a $\sim 250$ ps window with 2-3 
ps intrinsic time resolution ${ }^{25}$. In this work, an additional moving window average is applied to the data, reducing the time resolution to $10 \mathrm{ps}$. Time resolution is obtained by spectrally resolving interference between the two probes in a way that is analogous to ultrafast time resolution shock characterization methods used previously ${ }^{21,26,27}$.

A schematic of the pump-probe geometry and a picture of the sample region in a DAC after an experiment is shown in fig. Error: Reference source not found. This experimental setup is similar to that used in previous work ${ }^{20,28}$, but in this case using chirped pulses. The surface of the sample is imaged at a numerical aperture of 0.3 with a total magnification of $\sim 10 \mathrm{x}$ onto the slit of a $0.3 \mathrm{~m}$ imaging spectrometer (Acton 2300i) with a $1800 \mathrm{~g} / \mathrm{mm}$ grating, with the probe and pump profiles centered on the spectrometer slit. The slit width is typically $\sim 20 \mu \mathrm{m}$, corresponding to the CCD pixel width and a $2 \mu \mathrm{m}$ width at the sample plane. The CCD (a Princeton Instruments Pixis 100) is peltier-cooled to $-75 \mathrm{C}$, with $1340 \times 10016$ bit, $20 \times 20 \mu \mathrm{m}^{2}$ pixels. At $800 \mathrm{~nm}$, the CCD has a spectral range of $\sim 22 \mathrm{~nm}$. The pump is focused with a relatively high numerical aperture of $\sim 0.1$ to avoid self-focusing before reaching the ablator. The pump waist is typically 200-500 $\mu \mathrm{m}$ downstream from the ablator to achieve a 20-50 $\mu \mathrm{m}$ pump spot size (FWHM intensity) at the ablator. 

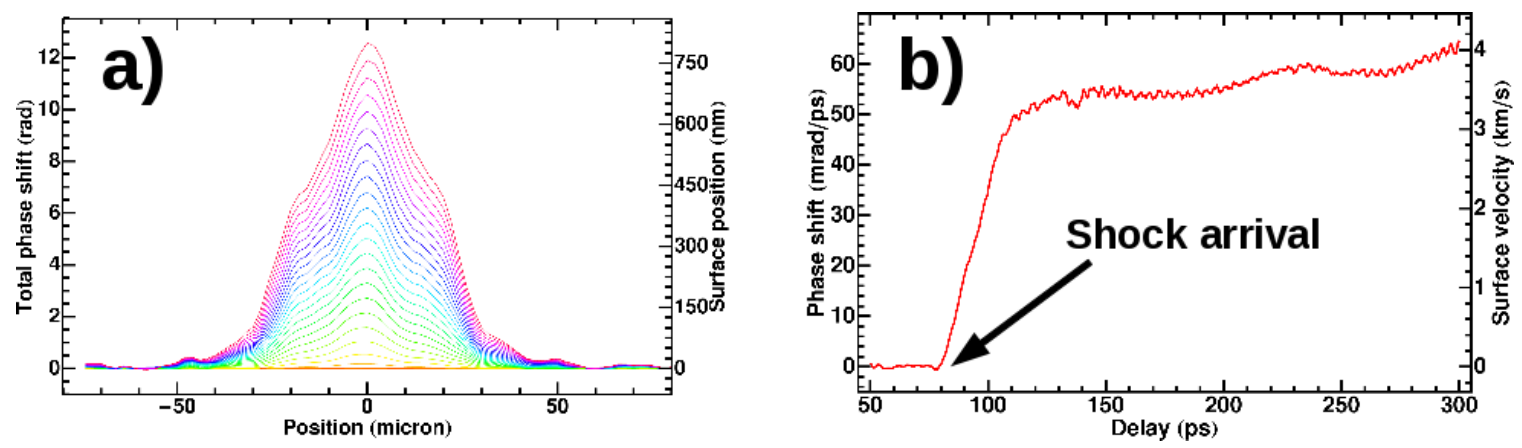

Figure 4: a) A measured breakout profile for a shocked, free aluminum surface expanding into air. These curves were generated in a single shot. Surface displacement is assumed to be proportional to the integrated finite derivative of the probe phase, as described in the text. Adjacent contours are separated by $10 \mathrm{ps}$ of surface motion. Later curves are taller than earlier curves. b) The finite derivative of the phase of the probe vs. delay measured at the peak of the breakout profile. This data is directly analogous to the phase shift measured by traditional VISAR diagnostics and is proportional to the surface velocity. Assuming a particle velocity of $1 / 2$ the surface velocity and the known Hugoniot of aluminum, we estimate the peak shock pressure for this experiment to be $\sim 37$ GPa.

A 10 ps time delay between the two probe pulses results in intensity beating in the spectrum, with $\sim 100$ fringes over the spectral range of the spectrometer. This intensity beating records a time dependent phase shift between the two probe pulses which occurs during the 10 ps delay between the probes. The phase of the intensity profile is recovered using the method of Kim et al. ${ }^{29}$ and the phase shift due to shock wave injection into the sample is obtained by taking the phase shift difference between pumped and unpumped regions of the sample compared to the phase background of the probe alone, analogous to the method of Gahagan et al. ${ }^{28}$

The method of this work measures a finite difference of the phase of reflected probe light accumulated (via motion of the ablator/sample interface or the shock front) over the probe pair time delay. When this phase difference is divided by the probe pair delay, the result is a finite derivative of the phase of the reflected probe. Successive values for the measured finite difference, spaced by the probe pair delay, may be summed to obtain the integrated phase, which is proportional to the surface position for shock breakout of a free surface. An example of the integrated finite difference of the 
phase vs. position along the slit and the finite derivative of the phase vs. time at the peak of a shock breakout at an aluminum/air interface is shown in fig. 4. In the case of a metal surface expanding into vacuum (i.e. when the probe does not traverse shocked material), the profile shown in fig. $4 \mathrm{~b}(\Delta \phi / \Delta \mathrm{t})$ gives the surface velocity via the same expression as used in VISAR ${ }^{24}$, i.e.,

$$
\text { surface velocity }=\frac{\lambda}{4 \pi} \frac{\Delta \phi}{\Delta t}
$$

where $\lambda$ is the probe wavelength and $\Delta \mathrm{t}$ is the probe pair delay. The data of fig. $4 \mathrm{~b}$ are directly analogous to VISAR measurements taken on longer time scales.

Since the time scale of the experiment is relatively short (100s of ps), the pump is focused to a small spot size without compromising the 1D planar shock wave approximation. In particular, the shock breakout profiles shown in fig. 4a are self-similar subsequent to shock arrival, indicating that shock curvature does not play a strong role in the shock propagation over the time scale of the experiment. We estimate the shock curvature of the data in fig. 4 a to be greater than $2 \mathrm{~mm}$ - sufficiently large compared with the propagation distance (which, on the time scale of the probe, was approximately one micron) to assume 1D wave propagation. 

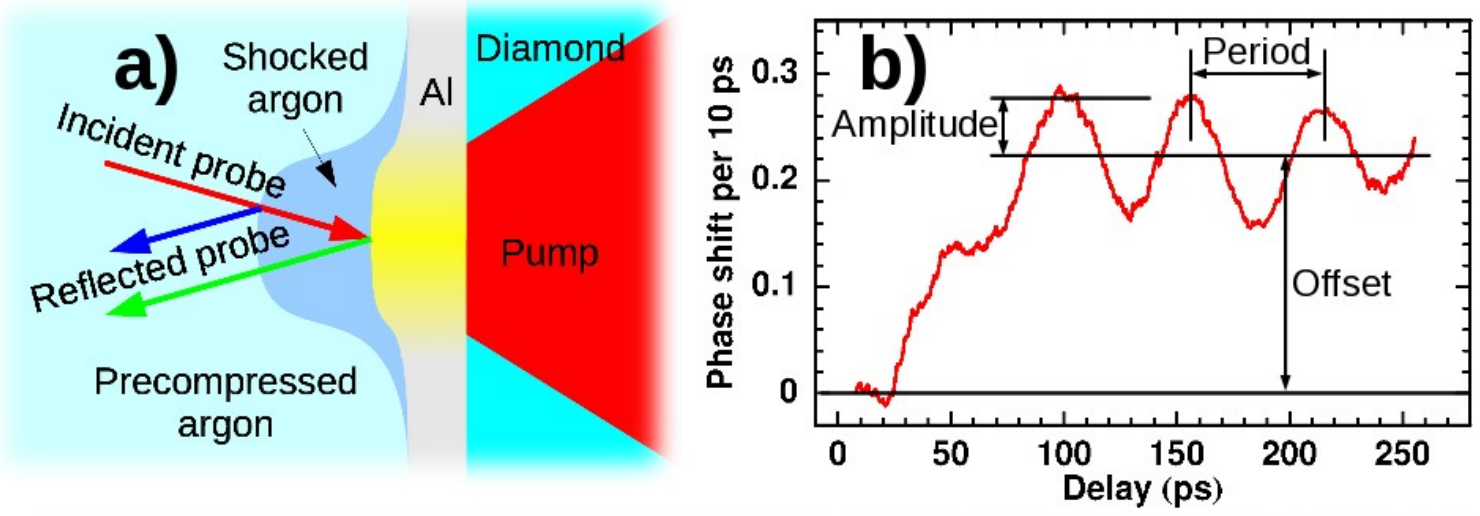

c)

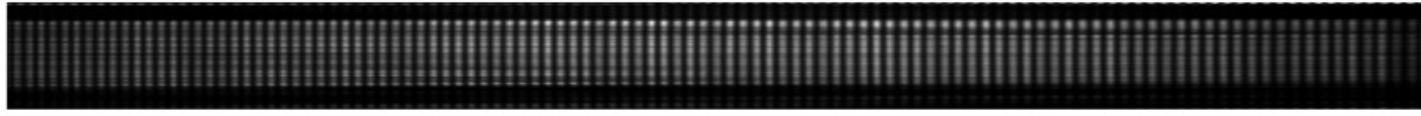

d)

Figure 5: a) A schematic of the probe interaction with the shocked region. Although only the first probe reflection from the shock front is shown, the shocked region is a dielectric film with multiple internal reflections of the probe. For a sufficiently small index rise at the shock front ( $<0.9$ for compressed argon with a precompressed index of $\sim 1.4$ ), only the first order reflections need be considered. The pump diameter is much larger than the distance the shock wave travels over the duration of the experiment, b) An example of the shock induced phase shift data in argon precompressed to $7.8 \mathrm{GPa}$, with a description of the parameterization, $c$ ) The raw spectral data with the probe pulses only, corresponding to $\sim 22 \mathrm{~nm}$ of bandwidth centered at $800 \mathrm{~nm}$. Longer wavelengths are to the right. The probe pulse is chirped so that red wavelengths arrive at the sample earlier than blue wavelengths, so time in the raw spectral data runs from right to left, d) the raw spectral data with the pump. Light near the vertical center of the trace corresponds to the pump spatial position.

Also, consistent with previous work, we assume steady wave propagation. In particular, because this method measures a finite difference of the phase, phase shifts resulting from index of refraction variation in the sample or the ablator do not contribute to the signal, since these signal contributions are constant for a steady wave. The nearly constant offset shown in fig. $4 \mathrm{~b}$ demonstrates that this method generates shock waves which are sufficiently steady for our analysis.

When a transparent material is shocked, as shown schematically in fig. 5a, the shock front acts as a moving dielectric mirror, partially reflecting and Doppler shifting probe light. The moving metal ablation layer also Doppler shifts reflected probe light, 
and optical beating between these two reflections results in a high frequency $(>10 \mathrm{GHz})$ oscillation in the phase, which is not detected in VISAR experiments (which typically have greater than 100 ps time resolution). An example of raw spectral data is shown in figs. 5c and 5d, and phase shift data obtained from argon shocked from an initial pressure of $7.8 \mathrm{GPa}$ is shown in fig. 5b. These data may be described by an offset added to a sinusoidal oscillation. Because the probe now traverses a shocked region with a different index of refraction than the unshocked region, the phase offset now includes a contribution from the advancing shock front in addition to surface motion of the ablator.

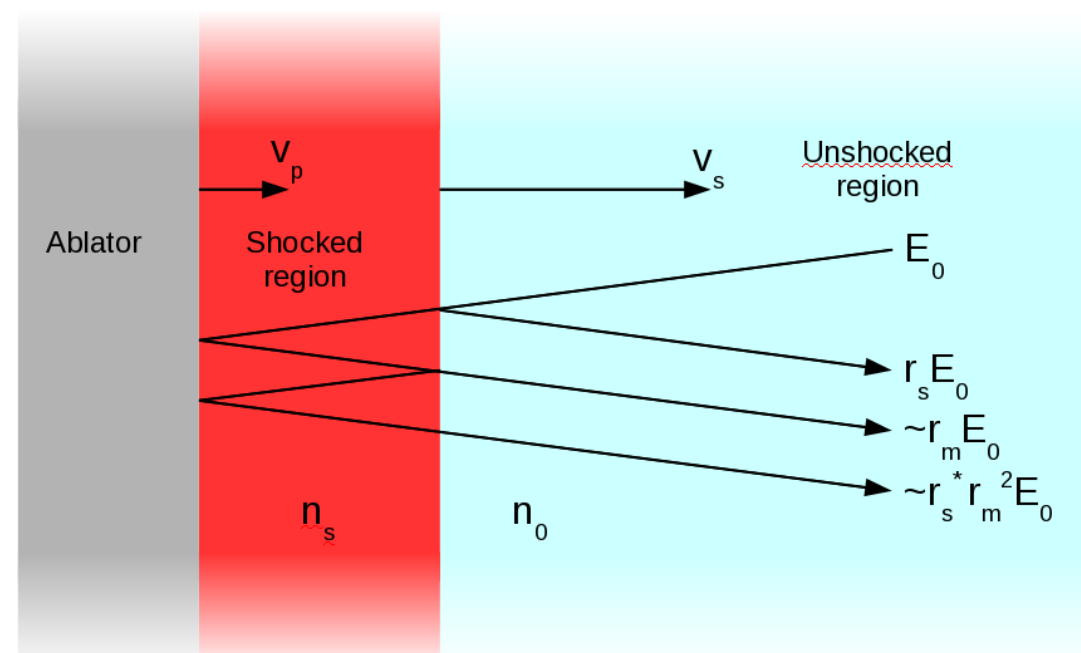

Figure 6: Schematic of the model. The incident probe $\left(E_{0}\right)$ is reflected from the shock front and the ablation layer. Only first order terms in $r_{s}$ are included in the approximation of the reflectivity. The particle velocity is assumed to be the same as the velocity of the interface between the ablator and the shocked sample. We assume the shocked refractive index is larger than the unshocked index. The reflectivity of the ablator $\left(r_{m}\right)$ is close to 1 .

Figure 6 illustrates the model. For a small index rise at the shock front, the total reflectivity amplitude will comprise two parts. A larger amplitude part reflects from the ablator, but not the shock front (with a reflectivity amplitude of $\sim r_{m}$, which we refer to as the ablator part). A smaller amplitude part comprises two first order reflections from 
the shock front (with reflectivity amplitudes of $r_{s}$ and $\sim r_{s}^{*} r_{m}^{2}$, which we refer to as the shock part). Analogous to the linearly varying phase (i.e. constant phase derivative) of the single interface experiment shown in fig. 4, the ablator part will have a phase of:

$$
\text { phase of ablator part }=\frac{4 \pi}{\lambda}\left(n_{s} v_{p}-\Delta n v_{s}\right) t \text {, }
$$

which corresponds to the optical path through the shocked region with no reflections at the shock front, where $t$ is time after shock arrival and other terms are defined in fig. 6 . The time derivative of eq. 2 for a shocked transparent region is analogous to eq. 1 for the single interface. The derivative of this part of the reflected phase gives the constant offset shown in fig. $5 b$.

Also, the shock front and metallic ablator surface act as a scanning optical etalon, with a total reflectivity that varies periodically with the thickness of the shocked region, with the spatial period: $\frac{\lambda}{2 n_{s}}$. The thickness of the etalon as a function of time is given by $\left(v_{s}-v_{p}\right) t$. As, via shock front and ablator motion, the optical path through the etalon sweeps through one spatial period, the total reflectivity of the shocked region varies sinusoidally in both amplitude and phase with a period $\tau$ given by:

$$
\tau\left(v_{s}-v_{p}\right)=\frac{\lambda}{2 n_{s}} .
$$

Eq. 3 is analogous to the expression used to determine the speed of sound in time domain reflectivity measurements of acoustic waves ${ }^{20,31,32}$.

The shocked index of refraction can be determined from the amplitude of the phase oscillation shown in fig 5b. We derive this connection in detail below and only 
summarize here. Since the reflectivity amplitude for the entire shocked region is the sum of a large contribution (from the ablator reflection) and a small phase shifted contribution (from the shock reflections), it will have the form $A\left[1+\delta_{\max } \exp \left(\frac{2 \pi i}{\tau} t\right)\right]$, where A is constant, $\delta_{\max }$ depends on the reflectivity of the shock front and the ablator and is much less than one, and $\tau$ is the period of the scanning etalon formed by the shock front and the ablator. The phase of the total reflectivity amplitude will vary sinusoidally with time, i.e.,

$$
\begin{aligned}
\operatorname{Arg}\left[A\left(1+\delta_{\max } \exp \left(\frac{2 \pi i}{\tau} t\right)\right)\right] & \approx \operatorname{Arg}\left[\operatorname{Aexp}\left(\delta_{\max } \exp \left(\frac{2 \pi i}{\tau} t\right)\right)\right] \\
& =\delta_{\max } \sin \left[\frac{2 \pi}{\tau} t+\phi\right]
\end{aligned}
$$

where $\phi$ is related to the phase of the reflectivity of the shock front and ablator. As we show below, it is possible to determine $r_{s}$ (and thus $n_{s}$, the shocked index of refraction) knowing $\delta_{\max }$, which is related to the measured oscillation amplitude. Given the shocked index of refraction, eqs. 2 and 3, and the unshocked index of refraction, the parameters of fig. 5b determine the shock and particle velocities.

To demonstrate this from first principles, we expand the optical reflectivity of the shocked region to first order in terms of the reflectivity of the shock front, which is assumed to be a thin optical interface (i.e. the probe wavelength is much larger than the longitudinal extent of the shock front, consistent with previous work ${ }^{21}$ ). For a probe at 
normal incidence, the total reflectivity, $\mathrm{R}$, of the thin film structure formed by the shock region and illustrated in fig. 6 is:

$$
R=-r_{s}+\frac{\left(1-\left|r_{s}\right|^{2}\right) r_{m}}{1-r_{m} r_{s}^{*}}
$$

where $r_{m}$ and $r_{s}$ are defined below and the second term is the result of summation over all internal reflections. Eq. 5 explicitly accounts for optical reciprocity at the shock front, where $\operatorname{Arg}[t]=\operatorname{Arg}\left[t^{\prime}\right]=0$ to first order in $\quad r_{s}$, and $\quad r_{s}{ }^{\prime}=-r_{s}{ }^{*} \quad$ consistent with the formalism of Ou and Mandel ${ }^{30}$. We assume $r_{m}$ and $r_{s}$ include phase shifts due to interface motion and the index of refraction increase behind the shock front as follows:

$$
\begin{array}{cc}
r_{s}=r_{s 0} \exp \left[i \theta_{s}\right] & \theta_{s}=\frac{4 \pi}{\lambda} n_{0} v_{s} t \\
r_{m}=r_{m 0} \exp \left[i \theta_{m}\right] & \theta_{m}=\frac{4 \pi}{\lambda}\left(n_{s} v_{p}-\Delta n v_{s}\right) t
\end{array}
$$

where $v_{s}$ is the shock velocity, $v_{p}$ is the particle velocity, $\lambda$ is the probe wavelength in vacuum, $n_{s}$ is the index of refraction of the shocked region, $t$ is elapsed time after the shock arrival, and $r_{s 0}, r_{m 0}$ are the electric field reflectivities of the shock and ablator interfaces, respectively. Here $r_{s 0}, r_{m 0}$ are assumed constant over the duration of the experiment (after shock arrival), consistent with a steady wave. The phase shifts $\theta_{s}, \theta_{m}$ result from motion of the shock and ablator interfaces, and the increase in the index of refraction behind the shock front. Expanding the total reflectivity (eq. 5) to first order in $r_{s}$ gives: 


$$
\begin{aligned}
R & \approx-r_{s}+r_{m}+r_{m}^{2} r_{s}^{*} \\
& =\left(-r_{s 0} \exp \left[i\left(\theta_{s}-\theta_{m}\right)\right]+r_{m 0}+r_{m 0}^{2} r_{s 0}^{*} \exp \left[-i\left(\theta_{s}-\theta_{m}\right)\right]\right) \exp \left[i \theta_{m}\right]
\end{aligned}
$$

A sketch of reflectivities in the complex plane is shown in fig. 7. Although there are two terms proportional to $r_{s}$ in eq. 7 , these terms are symmetrical with respect to a perpendicular to $r_{m}$, as shown in fig. 7 and discussed below. The phase of the reflected probe differs from the acoustic situation (detailed in Lin et al. ${ }^{32}$ ) primarily in that the total phase has a linear variation in addition to the sinusoidal variation found in the acoustic reflectivity. This additional linear variation in the phase of the reflectivity is the qualitative signature of a shock wave. The phase of the total reflectivity $(\mathrm{R})$ is:

$$
\theta=\theta_{m}+\delta
$$

A finite difference of the phase over the probe pair delay is measured, i.e.,

$$
\Delta \theta=\Delta \theta_{m}+\Delta \delta
$$

where $\Delta \theta$ is the measured signal shown in fig. $5 \mathrm{~b}$ and $\delta$ is defined in fig. 7. First, we note that $\Delta \theta_{m}$ corresponds to the measured phase offset. In particular,

$$
\text { offset }=\frac{\Delta \theta_{m}}{\Delta t} \approx \frac{d \theta_{m}}{d t}=\frac{4 \pi}{\lambda}\left(n_{s} v_{p}-\Delta n v_{s}\right)
$$


from eq. 6 above. Next, the phase of terms proportional to $r_{\text {so }} \quad$ (in parenthesis) in eq. 7 is

$$
\left|\theta_{m}-\theta_{s}\right|=\frac{4 \pi}{\lambda}\left(v_{s}-v_{p}\right) n_{s} t
$$

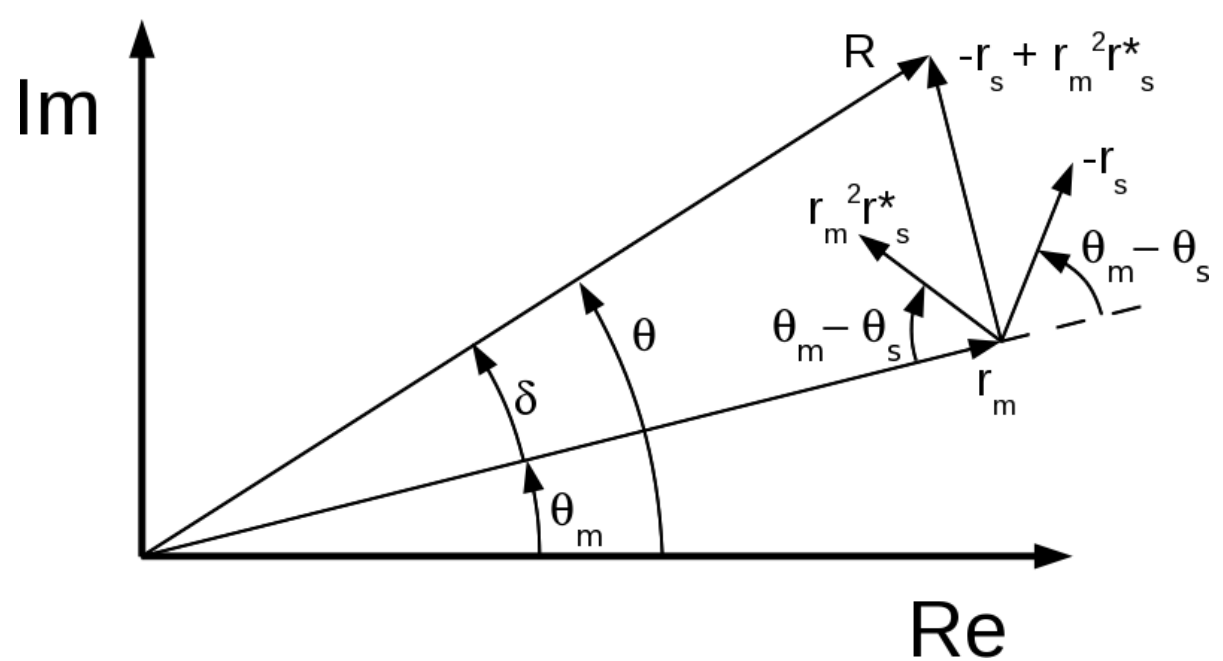

Figure 7: Reflectivities for terms in eq. 7 in the complex plane. Reflectivities corresponding to $\mathbf{r}_{\mathrm{m}}{ }^{2} \mathbf{r}_{\mathrm{s}}$ and $-r_{s}$ rotate in opposite directions and are symmetric about a perpendicular to $r_{m}$. The phase which corresponds to oscillations in the signal, $\delta$, is at a maximum or minimum when $r_{\mathrm{m}}{ }^{2} \mathbf{r}_{\mathrm{s}}$ and $-\mathbf{r}_{\mathrm{s}}$ are parallel. In the acoustic wave case, $r_{m}$ remains stationary while the sum $r_{m}{ }^{2} r_{s}{ }_{s}-r_{s}$ oscillates sinusoidally along a perpendicular to $r_{m}$. In the shock case, the reflectivities all rotate with the time dependence of $\theta_{m}$.

Assuming a single cycle of phase variation (of $\theta_{m}-\theta_{s}$ ) is completed over a period of $\tau$, this relation gives:

$$
v_{s}-v_{p}=\frac{\lambda}{2 n_{s} \tau}
$$

where $\tau$ is the measured period of the oscillations shown in fig. 5b, consistent with work in picosecond acoustics ${ }^{20,31,32}$. Finally, referring to fig. 7, we note that, for $r_{m} \approx 1$, terms in eq. 7 that are proportional to $r_{s}$ will sum to a reflectivity that is approximately perpendicular to $r_{m}$ in the complex plane, and identically perpendicular to $r_{m}$ for extrema (i.e. when terms proportional to $r_{s}$ sum to a maximum 
amplitude), independent of the magnitude of $r_{m}$. So, when $\delta$ is at a maximum,

$$
\begin{aligned}
\left|-r_{s}+r_{m}^{2} r_{s}^{*}\right|=\left|r_{s}\right|\left(1+\left|r_{m}\right|^{2}\right) & \text {, giving: } \\
\qquad \delta_{\max } & =\arctan \left[\frac{\left|r_{s}\right|\left(1+\left|r_{m}\right|^{2}\right)}{\left|r_{m}\right|}\right] \\
& \approx \frac{\left|r_{s}\right|\left(1+\left|r_{m}\right|^{2}\right)}{\left|r_{m}\right|}
\end{aligned}
$$

where $\delta_{\max }$ is the maximum value of $\delta$. For small $\left|r_{s}\right|$, we assume $\delta_{\max }$ is the same as the argument of the inverse tangent in eq. 13. Since the experiment measures a finite difference in phase, $\Delta \delta$, and, to a good approximation $\delta$ is a sinusoid of the form

$$
\delta=\delta_{\max } \sin \left[2 \pi \frac{t}{\tau}+\phi\right],
$$

where $\phi$ is the phase of the oscillation, then

$$
\begin{aligned}
\Delta \delta & \approx \frac{d \delta}{d t} \Delta t \\
& =2 \pi \frac{\Delta t}{\tau} \delta_{\max } \cos \left[2 \pi \frac{t}{\tau}+\phi\right]
\end{aligned}
$$

So,

$$
\delta_{\max }=\frac{\tau}{2 \pi} \frac{\Delta \delta_{\max }}{\Delta t}
$$

where $\Delta \delta_{\max }$ is the amplitude of the measured oscillations of the signal shown in fig. 5b. Assuming the index of refraction of the shocked material is real, the reflectivity and index of refraction rise at the shock front can be derived from

$$
\left|r_{s}\right|=\delta_{\max } \frac{\left|r_{m}\right|}{1+\left|r_{m}\right|^{2}} \quad \text { and } \quad \Delta n=\frac{2 n_{0}\left|r_{s}\right|}{1-\left|r_{s}\right|}
$$


where we assume $\left|r_{s}\right|=\frac{\Delta n}{2 n_{0}+\Delta n}$. In practice, $\frac{\left|r_{m}\right|}{1+\left|r_{m}\right|^{2}} \approx \frac{1}{2}$ and does not vary strongly with $\left|r_{m}\right|$ (which is typically close to 1 ), changing by only $2.4 \%$ between $\left|r_{m}\right|^{2}=1 \quad$ and $\quad\left|r_{m}\right|^{2}=0.64$

This derivation assumes that the reflectivity of the shock front is small, i.e. the first order approximation of the reflectivity (eq. 7), and the approximation of eq. 13. Nonetheless, these relations are accurate even for large index changes at the shock front. When simulations of the phase signal using the exact reflectivity (eq. 5) are used to derive the offset, period and oscillation amplitude, the above approximate relations give the correct index rise within $\sim 1.4 \%$ for $n_{0}=1.4$ and $\Delta n=0.9$, which (assuming a Gladstone-Dale relationship between the index of refraction and the density) corresponds to a change in density by more than a factor of 3 . We also assume that the derivative is well approximated by a finite difference in eqs. 10 and 15 . This approximation is exact for a constant offset and we estimate an error in the final pressure and density (from eq.

15) of less than $2 \%$ and $0.5 \%$, respectively. Finally, we assume that $\left|r_{m}\right|^{2}=1$, noting that a large change in $r_{m}$ is required to significantly change estimates of the parameters, and that, if need be, $\mathrm{r}_{\mathrm{m}}$ could be measured from the raw spectral data. Estimates of the period do not depend on approximations.

We assume the shocked material has a real index of refraction (i.e. is nonmetallic), but this assumption fails at sufficiently large shock pressures. Nonetheless, it may be possible to determine a complex index of refraction at the shock front from 
further analysis by taking into account the phase of the oscillation ( $\phi$ ) with respect to shock arrival.

In summary, given three measured parameters from the phase shift data (as shown in fig. 5b): the offset ( $\Delta \theta_{m}$ ), period ( $\tau$ ), and oscillation amplitude ( $\Delta \delta_{\max }$ ), we calculate the shock velocity, particle velocity, and index of refraction behind the shock front via the following relations:

$$
\begin{gathered}
\delta_{\max }=\frac{\tau}{2 \pi} \frac{\Delta \delta_{\max }}{\Delta t} \\
\left|r_{s}\right|=\delta_{\max } \frac{\left|r_{m}\right|}{1+\left|r_{m}\right|^{2}} \\
\Delta n=\frac{2 n_{0}\left|r_{s}\right|}{1-\left|r_{s}\right|} \\
n_{s}=n_{0}+\Delta n \\
v_{s}=\frac{\lambda}{4 \pi n_{0}}\left[\frac{\Delta \theta_{m}}{\Delta t}+\frac{2 \pi}{\tau}\right] \\
v_{p}=\frac{\lambda}{4 \pi n_{0}}\left[\frac{\Delta \theta_{m}}{\Delta t}+\frac{2 \pi}{\tau}\left(1-\frac{n_{0}}{n_{s}}\right)\right]
\end{gathered}
$$

where eqs. $18 \mathrm{e}$ and $18 \mathrm{f}$ follow from eqs. 12 and 10 . The above relations enable a straightforward connection between error in estimates of the parameters and error in the derived shock quantities. All of the above derivation applies to shock waves regardless of whether they propagate in a precompressed medium.

The data are analyzed by fitting to a quadratic function, which is then subtracted from the signal, leaving a sinusoid. The sinusoid is fitted to determine $\tau$ and $\Delta \delta_{\max }$ and an average of the quadratic function (which is mostly a constant) is taken to determine $\Delta \theta_{m}$. 
We do not observe etalon effects from the diamond/sample interface in the analyzed shock data. We attribute this to a loss of spatial coherence between subsequent internal reflections in the sample resulting from a slight tilt between the diamond/sample interface and the ablator/sample interface, and an imaging depth of field that is $2-3 \mathrm{x}$ shorter than the sample thickness (typically 20-30 $\mu \mathrm{m}$ ). Since the total propagation distance for shock waves in this study is $\sim 1 \mu$ m (i.e. much smaller than the depth of field), the reflectivity of the shocked region is accurately described by the plane wave formalism used above.

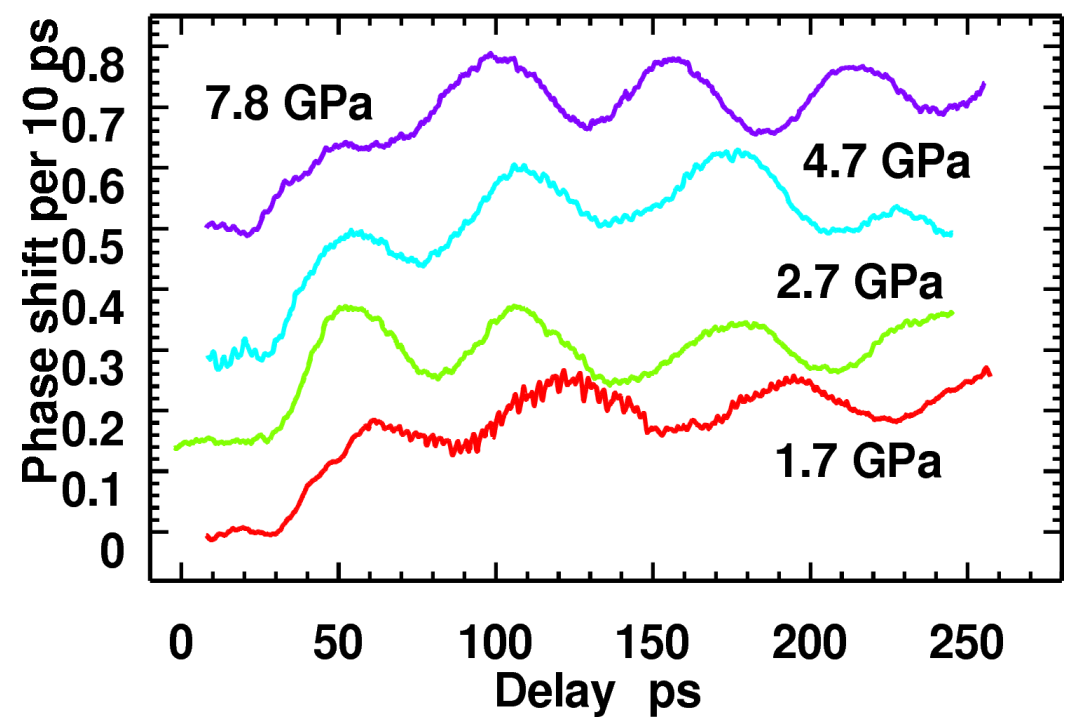

Figure 8: Shock induced phase shift data taken at precompressions labeled by initial pressure. The data are vertically offset for clarity.

\section{Results}

Raw phase shift data from argon shocked from initial pressures up to $7.8 \mathrm{GPa}$ are shown in fig. 8, with the data traces labeled by initial pressure. We assume the refractive index of shock compressed argon is real. Previous work with shocked liquid argon ${ }^{33}$ indicates that argon is not significantly electronically excited below a shock pressure of 40 GPa. Since states shocked from a higher initial density will have lower temperature 
for the same final shock pressure, and the shock pressures obtained in this study are less than $40 \mathrm{GPa}$, we do not expect significant electronic excitation in the shocked states generated for this work. As such, the index of refraction of shock compressed argon in this study should not have a significant imaginary component.

From the data of fig. 8, the offset, oscillation period and amplitude are determined, giving the shock velocity, particle velocity, and the index rise at the shock front. Then, given the initial pressure and density of the sample, the pressure and density of the shocked state are calculated via the usual methods of shock compression ${ }^{6}$, given by:

$$
\rho_{s}=\rho_{0} \frac{v_{s}}{v_{s}-v_{p}} \quad \text { and } \quad P_{s}=P_{0}+\rho_{0} v_{s} v_{p}
$$

where $P_{0}, \rho_{0}$ are the initial pressure and density, respectively, and $P_{s}, \rho_{s}$ are the shocked pressure and density, respectively.

A plot of the measured thermodynamic states of the shocked argon in pressuredensity space is shown in fig. 9. Also shown for comparison are the Hugoniot of liquid cryogenic argon $(84 \mathrm{~K})^{33}$, the solid argon room temperature isotherm ${ }^{16}$, and the calculated boundaries of the solid and liquid equilibrium phases of argon. The calculations were performed using fluid and solid variational theories ${ }^{34,35}$ with the exp-6 potential developed by $\operatorname{Ross}^{36}$, which reproduce very well the experimental Hugoniot, room temperature isotherm and melting line of argon. Except for the $2.7 \mathrm{GPa}$ data (which we discuss below), the parameters of the signal (offset, amplitude, and period) do not strongly vary, supporting the supposition of a steady shock wave over the duration of the experiment. 
We estimate the index of refraction of shocked states in the DAC to be 1.53 and 1.54 (7.8 GPa), 1.49 (4.6 GPa), 1.45 (2.7 GPa), and 1.41 and 1.40 (1.7 GPa), with an uncertainty of $1 \%$.

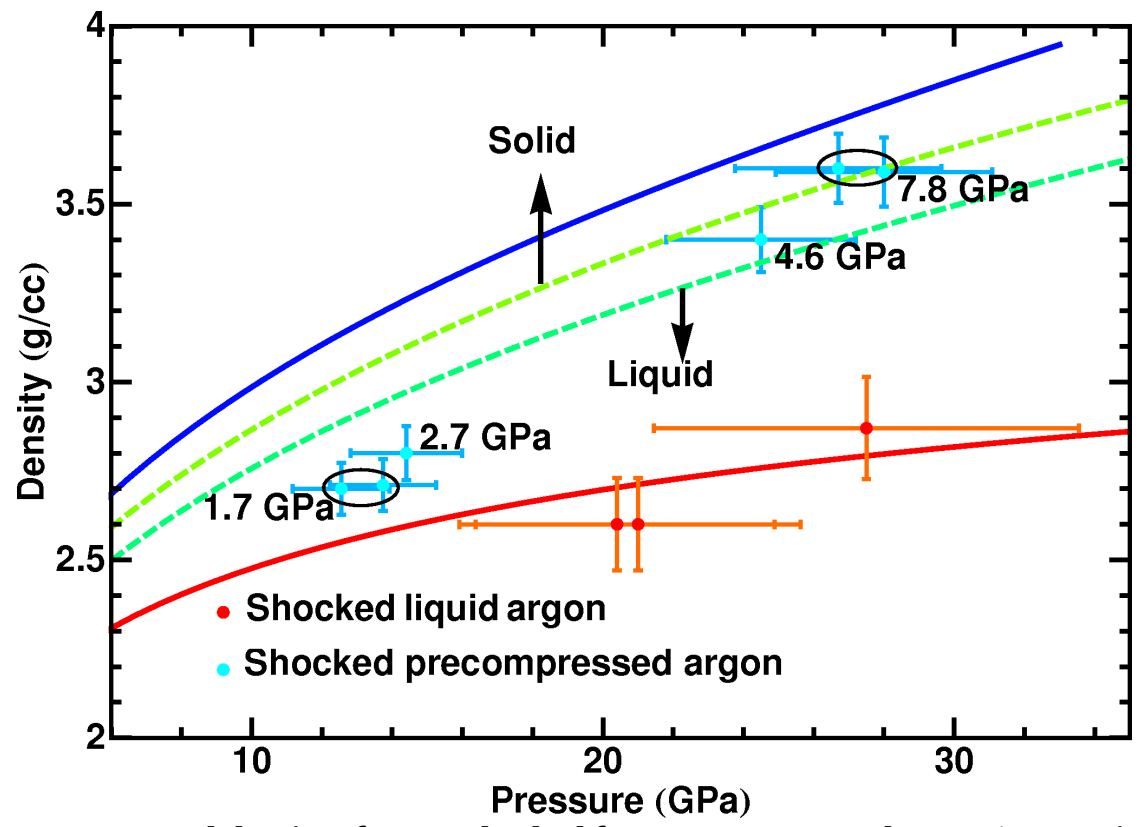

Figure 9: The pressure and density of argon shocked from precompressed states (cyan points) and from the liquid state at $84 \mathrm{~K}$ (red points). The precompressed points are labeled by the initial applied pressure. The curves are the cryogenic $(84 \mathrm{~K})$ liquid argon Hugoniot in red, the calculated boundaries of the equilibrium liquid and solid states in green, and the room temperature isotherm in blue. Argon at equilibrium is liquid at densities below the dashed melt line. The error bars indicate systematic error due to variation between the data and the model. The error for pressure is given by eq. 20 , and the error for density is given by an analogous expression for density. Scatter between points at the same precompression is due to shot to shot variation in laser power, variation of the thickness of the ablated film between shots, and alignment of the pump with the spectrometer slit. Nonetheless, all shots are estimates of states along the Hugnoiot for a given precompression.

We also obtained shock data from cryogenic liquid argon at $84 \mathrm{~K}$ in a custom

built dewar. The conditions under which data were obtained in the cryogen were significantly worse than the conditions under which data were obtained in the DAC, resulting from strong turbulence in the sample during the course of the experiment. Nonetheless, we obtain data which is sufficiently consistent with the Hugoniot derived from gas gun based measurements ${ }^{33}$ to demonstrate a significant variation between 
shocked states obtained under precompression compared to the cryogenic liquid Ar state at room pressure.

Error of all parameters over the record is estimated and included in the error bars for the final shock pressure and density. We assume a maximum error of $+/-15 \%$ in the estimate of the offset and oscillation amplitude, and a $+/-3 \%$ error in the estimate of the oscillation period for the precompressed data, and a $+/-30 \%$ error in the estimate of the offset and oscillation amplitude, and a $+/-6 \%$ error in the estimate of the period for the cryogenic liquid argon data. The larger uncertainty in the parameter estimates for the liquid argon data results from thermal fluctuations in liquid argon, which impacted the pump alignment with respect to the spectrometer slit. We estimate error in the pressure and density by directly evaluating the change in derived observables (shocked pressure, density and index) from variation between the data and the model, which assumes a constant offset plus a constant amplitude sinusoid, adding independent contributions in quadrature, e.g.

$$
\Delta P=\sqrt{\left(\frac{\partial P}{\partial \tau} \Delta \tau\right)^{2}+\left(\frac{\partial P}{\partial \delta_{\max }} \Delta \delta_{\max }\right)^{2}+\left(\frac{\partial P}{\partial\left(\frac{d \theta_{m}}{d t}\right)} \Delta\left(\frac{d \theta_{m}}{d t}\right)\right)^{2}}
$$

for the error in the estimate of pressure, with a similar expression for density. We use the worst case error bars given within the DAC data for all DAC data points. The error bars we use are larger than what is indicated by noise alone. Primarily, the error bars indicate an estimate of the systematic error resulting from deviation between the form of the measured data and the form of the model (a constant offset plus single frequency sinusoid). 
Generally, only 2-3 shots providing useful data are obtained from a single DAC load. Although, for a well prepared load, we almost always obtain useful data from the first shot in a given DAC load, subsequent shots suffer from collateral damage from previous shots and lower quality ablator film - we use the highest quality portion of the ablator for the first shot.

We assume the initial density and refractive index of argon given by Grimsditch et al. ${ }^{16}$, based on a measurement of the initial precompression via ruby fluorescence ${ }^{37}$. The aluminum ablation layer is fabricated by direct compression of aluminum between two diamonds, which flattens the aluminum via plastic deformation. The thickness of the aluminum is initially measured via interferometry, then using a known equation of state for aluminum, via the transit time of a picosecond acoustic wave launched by a subablation 100 fs pulse.

Precompression changes the final shocked state via two qualitative effects. First, as the density and sound speed of the precompressed argon increases, the shock pressure transmitted from the aluminum ablator into the argon increases via improved shock impedance matching. Second, as the initial precompression increases, the degree of heating from a given shock compression decreases ${ }^{15}$, increasing the shocked density. As precompression is increased, the Hugoniot approaches isentropic compression ${ }^{6,15}$. At constant pump fluence, we find a strong increase in shock pressure with precompression of argon, and an increase in density which approaches the room temperature isotherm for argon, shocked from a precompression of $7.8 \mathrm{GPa}$. 
As the precompression is decreased, the final shocked state decreases in density faster than the melt line, approaching the cryogenic liquid argon Hugoniot. For solid argon shocked from a precompression of $1.7 \mathrm{GPa}$ (argon at room temperature melts at 1.3 GPa), we observe a shocked density that is well below the density along the melt line. Although this may indicate that shocked argon has melted, we note the possibility that a transient metastable state may also occur ${ }^{7,17}$. Further, inspection of the corresponding 1.7 GPa phase shift data in fig. 8 indicates that a characteristic sinusoidal oscillation (consistent with shock propagation) has begun within about 50 ps after the shock arrival at the $\mathrm{Al} / \mathrm{Ar}$ interface, exhibiting a constant period consistent with steady propagation thereafter. Even if the shocked state of the $1.7 \mathrm{GPa}$ data is metastable, on the time scale of this experiment the constant period of the data indicates that the shock wave has reached a steady state within 50 ps after arrival in the argon.

We note that the generated shock states can be varied over a region of phase space that is not easily accessible to single shock or static compression experiments. In combination with sample preheating and variation of the pump fluence, it should be possible to explore a much wider region of the material phase space off the standard room pressure Hugoniot. Further, although the shock data obtained from an initial pressure of 2.7 GPa was analyzed assuming a constant oscillation period, the raw data exhibits a slight shift in period around $t=\sim 120$ ps. We note that this shift occurs at relatively low precompression, near the room temperature melting pressure of $1.3 \mathrm{GPa}$. This shift suggests that a phase transition may be occurring over the time scale of the experiment. Although such a shift may be difficult to interpret, further study of shocked 
argon near the phase transition pressure is warranted. Argon is an ideal material with which to study such transition, since it is straightforward to simulate and does not undergo chemistry.

We note that this measurement does not depend on the details of connecting optical spectra (i.e. linear absorption, Raman, etc.) to pressure or density, which is often complex. We obtain a direct estimate of density via a method analogous to shock wave methods used for decades, providing an unambiguous characterization of the pressure and density of the shocked thermodynamic state over a picosecond time scale. We also note the great potential of similar measurements to examine thermodynamic states on ultrafast time scales and sub-micron length scales, amenable to low power laser systems and compact experimental setups.

In conclusion, we have observed shocked states off the room pressure Hugoniot by applying ultrafast shock wave techniques to argon that has been precompressed in a conventional diamond anvil cell. We have also developed a straightforward method for analyzing ultrafast shock wave data. Although our analysis is currently limited to nonmetallic shock induced index of refraction changes of less than 0.9 (for an initial index of 1.4), with further development it should be possible to extend the analysis outside of this regime. Since this technique has ultrafast time resolution, it is possible to explore the early time dynamics of shock induced phase transitions and chemical reactions. By varying the initial pressure, we are able to significantly modulate the final shock temperature and pressure, greatly extending the thermodynamic phase space accessible to single shock experiments. 
We acknowledge useful discussions with L. Fried, E. Glascoe, C. Grant, E. Reed, H. Lorenzana, C. Bolme, S. Mcgrane, and J. Forbes. This work was performed under the auspices of the U.S. Department of Energy by Lawrence Livermore National Laboratory under Contract DE-AC52-07NA27344 and funded in part by the DTRA Advanced Energetics program. 


\section{References}

1 Crowhurst, J. C., Brown, J. M., Goncharov, A. F., and Jacobsen, S. D., Elasticity of (Mg,Fe)O through the spin transition of iron in the lower mantle. Science 319, 451 (2008).

2 Zaug, J. M., Abramson, E. H., Brown, J. M., and Slutsky, L. J., Sound Velocities in Olivine at Earth Mantle Pressures. Science 260, 1487 (1993).

3 Nellis, W. J., Weir, S. T., and Mitchell, A. C., Metallization and electrical conductivity of hydrogen in Jupiter. Science 273, 936 (1996).

$4 \quad$ Nna-Mvondo, D., Khare, B., Ishihara, T., and McKay, C. P., Experimental impact shock chemistry on planetary icy satellites. Icarus 194, 816 (2008).

$5 \quad$ McGrane, S. D., Moore, D. S., and Funk, D. J., Shock induced reaction observed via ultrafast infrared absorption in poly(vinyl nitrate) films. J. Phys. Chem. A 108, 9342 (2004).

Nellis, W. J., Dynamic compression of materials: metallization of fluid hydrogen at high pressures. Rep. Prog. Phys. 69, 1479 (2006).

$7 \quad$ Kalantar, D. H. et al., Direct observation of the alpha-epsilon transition in shockcompressed iron via nanosecond x-ray diffraction. Phys. Rev. Lett. 95, 075502 (2005).

8 Dlott, D. D., Thinking big (and small) about energetic materials. Mater. Sci. Technol. 22, 463 (2006).

$9 \quad$ Bassett, W. A., Diamond anvil cell, 50th birthday. High Pressure Research 29, CP5 (2009). 
Hemley, R. J., Effects of high pressure on molecules. Annu. Rev. Phys. Chem. 51, 763 (2000).

11 Grochala, W., Hoffmann, R., Feng, J., and Ashcroft, N. W., The chemical imagination at work in very tight places. Anew. Chem., Int. 46, 3620 (2007). Smith, R. L. and Fang, Z., Techniques, applications and future prospects of diamond anvil cells for studying supercritical water systems. J. Supercrit. Fluids 47, 431 (2009).

13 Schettino, V. et al., Chemical Reactions at Very High Pressure. Adv. Chem. Phys. 131, 105 (2005). Hoover, W. G., Structure of a Shock-Wave Front in a Liquid. Phys. Rev. Lett. 42, 1531 (1979). Jeanloz, R. et al., Achieving high-density states through shock-wave loading of precompressed samples. Proc. Natl. Acad. Sci. U. S. A. 104, 9172 (2007). Grimsditch, M., Loubeyre, P., and Polian, A., Brillouin-Scattering and 3-Body Forces in Argon at High-Pressures. Phys. Rev. B 33, 7192 (1986). Evidence for Electronic Bond Hardening in Gold. Science 323, 1033 (2009). Dlott, D. D., Ultrafast spectroscopy of shock waves in molecular materials. Annu. Rev. Phys. Chem. 50, 251 (1999).

19 Greenfield, M., McGrane, S. D., and Moore, D. S., Control of cis-Stilbene Photochemistry Using Shaped Ultraviolet Pulses. J. Phys. Chem. A 113, 2333 (2009). 
Armstrong, M. R., Crowhurst, J. C., Reed, E. J., and Zaug, J. M., Ultrafast high strain rate acoustic wave measurements at high static pressure in a diamond anvil cell. Appl. Phys. Lett. 92, 101930 (2008).

21 Bolme, C. A., McGrane, S. D., Moore, D. S., and Funk, D. J., Single shot measurements of laser driven shock waves using ultrafast dynamic ellipsometry. J. Appl. Phys. 102, 033513 (2007).

Evans, R. et al., Time- and space-resolved optical probing of femtosecond-laserdriven shock waves in aluminum. Phys. Rev. Lett. 77, 3359 (1996).

Manheimer, W. M., Colombant, D. G., and Gardner, J. H., Steady-State Planar Ablative Flow. Phys. Fluids 25, 1644 (1982).

Barker, L. M. and Hollenbach, R. E., Shock-Wave Studies of Pmma, Fused Silica, and Sapphire. J. Appl. Phys. 41, 4208 (1970).

Kim, K. Y. et al., Single-shot, interferometric, high-resolution, terahertz field diagnostic. Appl. Phys. Lett. 88, 041123 (2006).

Geindre, J. P., Audebert, P., Rebibo, S., and Gauthier, J. C., Single-shot spectral interferometry with chirped pulses. Opt. Lett. 26, 1612 (2001).

Benuzzi-Mounaix, A. et al., Chirped pulse reflectivity and frequency domain interferometry in laser driven shock experiments. Phys. Rev. E 60, R2488 (1999). Gahagan, K. T. et al., Measurement of shock wave rise times in metal thin films. Phys. Rev. Lett. 85, 3205 (2000).

Kim, K. Y., Alexeev, I., and Milchberg, H. M., Single-shot supercontinuum spectral interferometry. Appl. Phys. Lett. 81, 4124 (2002). 
Ou, Z. Y. and Mandel, L., Derivation of reciprocity relations for a beam splitter from energy balance. Am. J. Phys. 57, 66 (1989). Sohn, J. Y. et al., Nano seismology: acoustic shock wave generation and terahertz emission from InGaN/GaN structures. Compound Semiconductors 2001, 387 (2002).

Lin, H. N., Stoner, R. J., Maris, H. J., and Tauc, J., Phonon Attenuation and Velocity-Measurements in Transparent Materials by Picosecond Acoustic Interferometry. J. Appl. Phys. 69, 3816 (1991). Vanthiel, M. and Alder, B. J., Shock Compression of Argon. J. Chem. Phys. 44, 1056 (1966). Ross, M., High-Density Fluid-Perturbation Theory Based on an Inverse 12thPower Hard-Sphere Reference System. J. Chem. Phys. 71, 1567 (1979). Kang, H. S. and Ree, F. H., A Variational Theory of Classical Solids. J. Chem. Phys. 99, 2985 (1993). Ross, M., Mao, H. K., Bell, P. M., and Xu, J. A., The Equation of State of Dense Argon - a Comparison of Shock and Static Studies. J. Chem. Phys. 85, 1028 (1986).

37 Zha, C. S., Mao, H. K., and Hemley, R. J., Elasticity of MgO and a primary pressure scale to 55 GPa. Proc. Natl. Acad. Sci. U. S. A. 97, 13494 (2000). 


\section{Figure captions}

Figure 1: The experimental setup, a) shows the optical setup external to the DAC. Although the probe pulses are shown as separated for the purpose of illustration, they overlap temporally. b) shows a close up of a cross section of the DAC, where precompressed sample is shocked via ablation of a $\sim 1$ micron aluminum layer on the culet of the right side diamond. PBS is a polarizing beam splitter.

Figure 2: a) a schematic of the stretcher with a knife edge and the unblocked beam path of red and blue components of the spectrum. G labels the gratings, and $f$ is the focal length of the focusing optics. The knife edge blocks the spectrum, creating a fast rise in the intensity of the chirped pulse. b) shows a typical spectrum of the laser pulse after the amplifier.

Figure 3: a) A schematic of the pump and probe imaged at the spectrometer slit and b) an image of a DAC from the pump side after an experiment. In this case, the sample was nitromethane, which reacted during the experiment and produced a black product which filled the DAC sample cavity. Although the reaction in this experiment was pump induced, it was not obviously correlated with interferometric probe data within 250 ps after the pump, and may have occurred after the probe time window. The short black horizontal line segments are camera artifacts. 
Figure 4: a) A measured breakout profile for a shocked, free aluminum surface expanding into air. These curves were generated in a single shot. Surface displacement is assumed to be proportional to the integrated finite derivative of the probe phase, as described in the text. Adjacent contours are separated by 10 ps of surface motion. Later curves are taller than earlier curves. b) The finite derivative of the phase of the probe vs. delay measured at the peak of the breakout profile. This data is directly analogous to the phase shift measured by traditional VISAR diagnostics and is proportional to the surface velocity. Assuming a particle velocity of $1 / 2$ the surface velocity and the known Hugoniot of aluminum, we estimate the peak shock pressure for this experiment to be 37 GPa.

Figure 5: a) A schematic of the probe interaction with the shocked region. Although only the first probe reflection from the shock front is shown, the shocked region is a dielectric film with multiple internal reflections of the probe. For a sufficiently small index rise at the shock front ( $<0.9$ for compressed argon with a precompressed index of $\sim 1.4$ ), only the first order reflections need be considered. The pump diameter is much larger than the distance the shock wave travels over the duration of the experiment, b) An example of the shock induced phase shift data in argon precompressed to $7.8 \mathrm{GPa}$, with a description of the parameterization, c) The raw spectral data with the probe pulses only, corresponding to $22 \mathrm{~nm}$ of bandwidth centered at $800 \mathrm{~nm}$. Longer wavelengths are to the right. The probe pulse is chirped so that red wavelengths arrive at the sample earlier than blue wavelengths, so time in the raw spectral data runs from right to left, d) the raw spectral 
data with the pump. Light near the vertical center of the trace corresponds to the pump spatial position.

Figure 6: Schematic of the model. The incident probe $\left(E_{0}\right)$ is reflected from the shock front and the ablation layer. Only first order terms in $\mathrm{r}_{\mathrm{s}}$ are included in the approximation of the reflectivity. The particle velocity is assumed to be the same as the velocity of the interface between the ablator and the shocked sample. We assume the shocked refractive index is larger than the unshocked index. The reflectivity of the ablator $\left(r_{m}\right)$ is close to 1 .

Figure 7: Reflectivities for terms in eq. 7 in the complex plane. Reflectivities corresponding to $\mathrm{r}_{\mathrm{m}}{ }^{2} \mathrm{r}_{\mathrm{s}}$ and $-\mathrm{r}_{\mathrm{s}}$ rotate in opposite directions and are symmetric about a perpendicular to $\mathrm{r}_{\mathrm{m}}$. The phase which corresponds to oscillations in the signal, $\delta$, is at a maximum or minimum when $\mathrm{r}_{\mathrm{m}}{ }^{2} \mathrm{r}_{\mathrm{s}}$ and $-\mathrm{r}_{\mathrm{s}}$ are parallel. In the acoustic wave case, $\mathrm{r}_{\mathrm{m}}$ remains stationary while the sum $r_{m}^{2} r_{s}-r_{s}$ oscillates sinusoidally along a perpendicular to $\mathrm{r}_{\mathrm{m}}$. In the shock case, the reflectivities all rotate with the time dependence of $\theta_{m}$.

Figure 8: Shock induced phase shift data taken at precompressions labeled by initial pressure. The data are vertically offset for clarity.

Figure 9: The pressure and density of argon shocked from precompressed states (cyan points) and from the liquid state at $84 \mathrm{~K}$ (red points). The precompressed points are labeled by the initial applied pressure. The curves are the cryogenic (84 K) liquid argon 
Hugoniot in red, the calculated boundaries of the equilibrium liquid and solid states in green, and the room temperature isotherm in blue. Argon at equilibrium is liquid at densities below the dashed melt line. The error bars indicate systematic error due to variation between the data and the model. The error for pressure is given by eq. 20, and the error for density is given by an analogous expression for density. Scatter between points at the same precompression is due to shot to shot variation in laser power, variation of the thickness of the ablated film between shots, and alignment of the pump with the spectrometer slit. Nonetheless, all shots are estimates of states along the Hugnoiot for a given precompression. 